Check for updates

Cite this: Nanoscale Horiz., 2020, 5, 1355

Received 11th April 2020, Accepted 29th July 2020

DOI: $10.1039 / \mathrm{d} 0 \mathrm{nh} 00207 \mathrm{k}$

rsc.li/nanoscale-horizons

\title{
Ultrasmall Au nanoclusters for bioanalytical and biomedical applications: the undisclosed and neglected roles of ligands in determining the nanoclusters' catalytic activities
}

\author{
Da Chen (D) *a and Jinghong Li (D)*b
}

\begin{abstract}
Significantly different from conventional Au nanoparticles, ultrasmall Au nanoclusters (NCs) consisting of several to about a hundred Au atoms with a size below $2 \mathrm{~nm}$ exhibit a strong quantum confinement effect, and possess an intriguing molecular-like highest occupied molecular orbital (HOMO)-lowest unoccupied molecular orbital (LUMO) transition, quantized charging, intrinsic chirality, and special fluorescence properties, as well as high catalytic activities. In virtue of their unique molecular-like electronic structure, remarkable physicochemical properties, mild preparation conditions and good biocompatibility, Au NCs have been having a profound impact on bioanalytical and biomedical applications, such as biosensing, biological imaging, cell markers, drug delivery, photodynamic/ photothermal therapy, and biomedical toxicology. As an indispensable part of Au NCs, shell ligands not only stabilize and protect the structure of Au NCs, but also have an important influence on the structure and biocatalytic activities of Au NCs. Nevertheless, the effect of shell ligands on the biocatalytic activities of Au NCs has not been paid much attention or even ignored. In this Focus article, thus, the structure and biocatalytic activities of Au NCs are discussed from the perspective of the shell ligands. Particular emphasis is directed to the discussion and exploration of the undisclosed and neglected roles of shell ligands in the biocatalytic activities of Au NCs, which are of fundamental importance to the unraveling of charge transfer behaviors and biocatalytic processes of Au NCs. In addition, the future directions to explore the mechanism of shell ligands affecting the biocatalytic activities of Au NCs, such as surface ligand engineering of Au NCs, advanced surface/interface in situ characterization techniques, theoretical analysis, and the nanobiology of Au NCs, are also put forward.
\end{abstract}

\section{Introduction}

In recent years, the rapid development of nanotechnology has brought new opportunities to the field of biomedicine and biological analysis. Biotechnological techniques based on nanomaterials have significantly improved the detection sensitivity and specificity. In particular, gold nanoclusters (Au NCs) have attracted particular attention due to their unique optical, electrical and chemical properties. ${ }^{1,2}$ Au NCs typically consist of several to about a hundred $\mathrm{Au}$ atoms, ranging in size from a single atom to a nanocrystal, with nuclear clusters less than $2 \mathrm{~nm}$ in diameter. ${ }^{3}$ Since the size of Au NCs is similar to the Fermi wavelength of the electrons, the continuous state density

\footnotetext{
${ }^{a}$ College of Materials and Chemistry, China Jiliang University, Hangzhou 310018, Zhejiang, China.E-mail:dchen_80@hotmail.com

${ }^{b}$ Department of Chemistry, Key Laboratory of Bioorganic Phosphorus Chemistry \& Chemical Biology, Tsinghua University, Beijing 100084, China.

E-mail: jhli@mail.tsinghua.edu.cn
}

is decomposed into discrete energy levels, making them significantly different from ordinary gold nanoparticles (Au NPs) $\left(>2 \mathrm{~nm}\right.$ in diameter) and bulk gold materials (Fig. 1) ${ }^{4,5}$ in terms of their optical, chemical, and electrical properties. ${ }^{6,7}$ Thanks to the strong quantum confinement effect, Au NCs possess intriguing molecular-like highest occupied molecular orbital (HOMO)-lowest unoccupied molecular orbital (LUMO) transition, ${ }^{8,9}$ quantized charging, ${ }^{10}$ intrinsic chirality, ${ }^{11}$ and special fluorescence properties, ${ }^{12,13}$ as well as high catalytic activity. ${ }^{6,14}$ In virtue of their advantages ${ }^{15,16}$ including ultrasmall size, good fluorescence stability, fluorescence adjustability, large Stokes shift, mild preparation conditions, and biocompatibility, Au NCs have been widely studied in the bioanalytical and biomedical field, such as biosensing, biological imaging, cell markers, drug delivery, the detection of biological molecules (e.g., DNA, protein and enzyme), and photodynamic/photothermal therapy, with great application prospects. It is noteworthy that these bio-related applications are usually inseparable from the biocatalytic activities of Au NCs. Different from industrial 


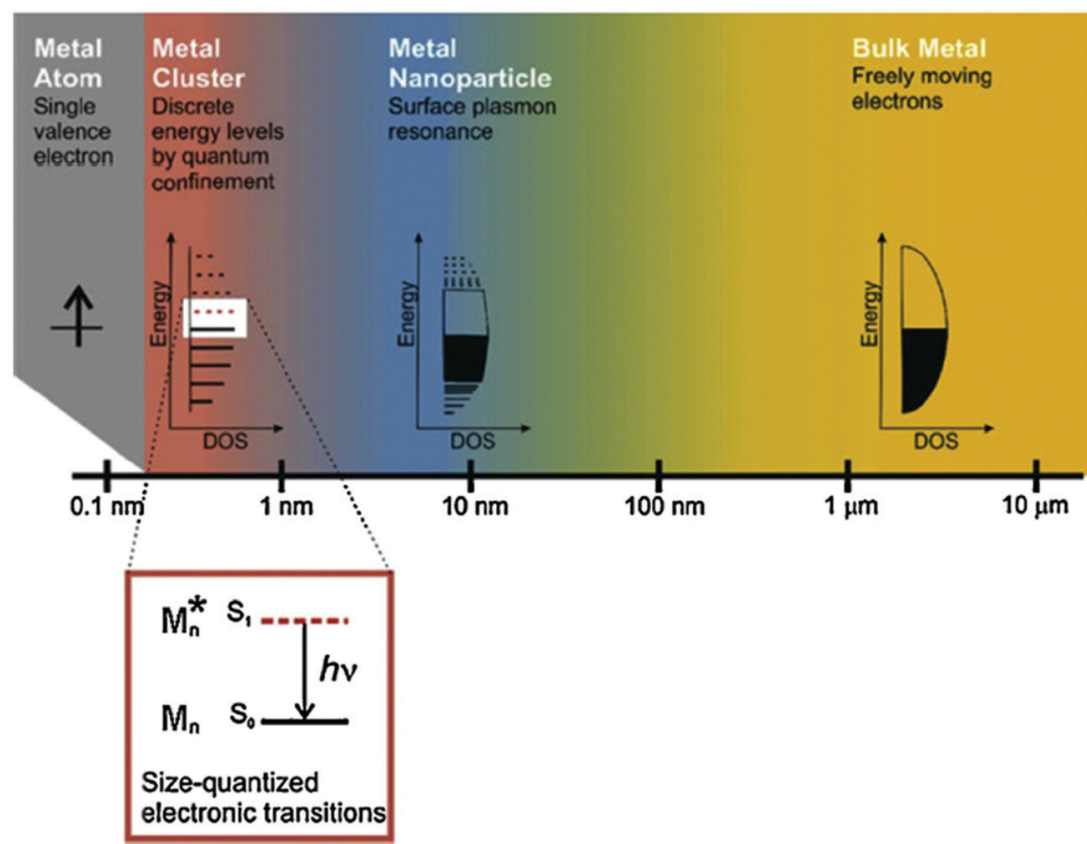

Fig. 1 The effect of size on metals. Whereas bulk metal and metal nanoparticles have a continuous band of energy levels, the limited number of atoms in metal nanoclusters results in discrete energy levels, allowing interaction with light by electronic transitions between energy levels. Metal nanoclusters bridge the gap between single atoms and nanoparticles. Reprinted from ref. 4 with permission. Copyright 2010 Springer-Verlag.

biocatalysis, ${ }^{17}$ nevertheless, the biocatalytic activities of Au NCs in this Focus article refers to the abilities of Au NCs to speed up (catalyze) the biochemical reaction processes in a variety of biological environments.

In view of the fact that $\mathrm{Au}$ is a chemically stable noble metal, it was not considered to have biocatalytic activity in the early stage. With the emergence of Au NPs, especially Au NCs, ${ }^{10,18}$ the biocatalytic activity of $\mathrm{Au}$ has attracted extensive attention in the field of biology and has made considerable research progress. At present, the biocatalytic activity of $\mathrm{Au}$ NCs is mainly reflected in the following aspects. Firstly, the unique fluorescence phenomenon generated by its quantum confinement effect $\mathrm{t}^{9,19-22}$ is used as a novel fluorescent probe for biomolecular detection, biological imaging and cell identification. Under a specific biological environment, the enrichment of Au NCs and the biochemical reactions involved in $\mathrm{Au}$ NCs will significantly affect the fluorescence intensity of Au NCs. Therefore, a change in fluorescence intensity of Au NCs can reflect to some extent the biochemical reactions in which $\mathrm{Au}$ NCs are involved. The second is to use its unique core-shell structure consisting of a core of zero-valent gold atoms $\left(\mathrm{Au}^{0}\right)$ and a shell of monovalent gold ions $\left(\mathrm{Au}^{+}\right)$to promote the process of charge transfer. ${ }^{23-25}$ The rapid charge transfer process within the core-shell structure of Au NCs can effectively promote the biochemical reactions between $\mathrm{Au}$ NCs and the surrounding biological components, which would further facilitate the recognition and detection of target analyte molecules, thus leading to the improvement of the sensitivity and selectivity of biosensor technology. Thirdly, under specific conditions, $\mathrm{Au}$ NCs also have certain enzyme-like catalytic activity, ${ }^{26,27}$ which can simulate some reactions catalyzed by biological enzymes.
In such cases, Au NCs usually participate directly in biocatalytic reactions. For example, gold nanoclusters stabilized using lysozyme show oxidase activity, which is expected to become a new substitute of peroxidase in the field of biological analysis. ${ }^{26}$ Lastly and most importantly, Au NCs produce photoacoustic or photothermal effects due to the occurrence of fluorescence resonance energy transfer (FRET) when contacting with some functional structures or molecules. ${ }^{16,28,29}$ Following light absorption, the photoacoustic or photothermal effects produced from Au NCs are generally related to the photophysical processes and photochemical reactions, in which some key processes, such as photon absorption and conversion of the absorbed pulsed or modulated radiation into phonons or heat energy, are accomplished through Au NCs. These effects have been widely used for photoacoustic imaging and photodynamic or photothermal therapy in the bioanalytical and biomedical fields especially in oncology. ${ }^{30}$ For example, Cui et $a .^{28}$ developed Au NCindocyanine green (ICG) nanoprobes by the conjugation of $\mathrm{Au}$ NCs with ICG for combined photoacoustic tumor imaging, therapy, and therapeutic monitoring in real time based on FRET. As is known, due to the quantum-size effect, "bare" Au NCs have very active chemical properties, which require the use of chemically synthesized molecules or biomolecules as ligands to stabilize and protect their surfaces, thus avoiding the aggregation of NCs and the loss of surface activity. ${ }^{14}$ Apparently, the biocatalytic activities of Au NCs are significantly affected by the size of the NCs, and the types and structural characteristics of the surface ligands.

However, it is worth paying attention to the point, that is, in the process of studying the biocatalytic properties of the atomically precise $\mathrm{Au}$ NCs, many researchers generally focus on the 
catalytic activities of $\mathrm{Au}$ NCs themselves, but often ignore the role of the ligands in the biocatalytic activities of $\mathrm{Au}$ NCs. In fact, the ligands have important impacts on the structural features of the NCs and have been also revealed to play an important role in other catalytic processes of $\mathrm{Au} \mathrm{NCs}^{31,32}$ including thermal catalysis, ${ }^{33,34}$ photocatalysis ${ }^{35,36}$ and electrocatalysis. ${ }^{37,38}$ Unlike the other catalytic reactions that require external factors (e.g., heat for thermal catalysis, light for photocatalysis, and electric fields for electrocatalysis), the biocatalytic process of Au NCs usually requires no external factors and involves a biochemical reaction that takes place in a specific biological environment. Due to the lack of external factors, the biocatalytic process of Au NCs is relatively difficult to occur and is more easily influenced by the shell structure of Au NCs (e.g., the hydrophilicity, the biocompatibility, the charge transfer properties, and the stability of the ligands). Thus, the biocatalytic activities of Au NCs are closely related to the ligands on the shell structure of NCs. Unfortunately, this correlation has not been paid attention to or even ignored. In this Focus article, we will discuss the biocatalytic activities of $\mathrm{Au}$ nanoclusters from the perspective of ligands, and put forward some personal viewpoints.

\section{Why atomically precise Au nanoclusters?}

\subsection{Are atomically precise nanoclusters really superior to conventional nanoparticles?}

From the existing research findings, it can be deduced that the biocatalytic activity of Au NCs is significantly better than that of conventional $\mathrm{Au}$ NPs. In terms of reactivity, Au NCs, with an ultrasmall particle size $(<2 \mathrm{~nm})$, possess much larger specific surface areas than Au NPs, and thus more active sites are present in NCs. Benefiting from the high surface binding energy of Au NCs, these active sites are more likely to adsorb and bind to the surrounding reactants, which would be favorable for the biocatalytic process. ${ }^{39}$ In relation to Au NPs, Au NCs exhibit specific fluorescence properties, better water solubility and biocompatibility, which makes Au NCs more likely to react with the substances in the surrounding biological environment for the biocatalytic process. This means that Au NCs are more suited for biological analysis, biological diagnosis and therapy than Au NPs. From the perspective of biological toxicity, Au NPs probably produce severe toxicity and side effects due to their accumulation in the liver and spleen, while Au NCs can be effectively removed from the body through renal clearance with no obvious toxicity and side effects to the human body. ${ }^{40-42}$ In addition, catalytic reactions usually occur on the catalyst surface, where the reactant atoms interact with each other at the active sites for the catalytic process. Since Au NCs are ultrasmall nanoclusters composed of a small number of $\mathrm{Au}$ atoms, from the perspective of fundamental mechanism understanding, they are supposed to be the ideal research unit for exploring the biocatalytic mechanism of Au both in theoretical simulation and experimental methods, ${ }^{43}$ which would help to deepen the understanding of the biocatalytic reaction processes and intrinsic mechanism of $\mathrm{Au}$ from the atomic scale. Last but not least, in terms of cost performance, the use of Au NCs can not only achieve higher catalytic activity than Au NPs, but also reduce the amount of precious metal Au to a large extent, thus greatly reducing the application cost. ${ }^{44}$

Apparently, Au NCs exhibit superior biocatalytic activities, although there are still many problems to be solved, such as the precise regulation of $\mathrm{Au}$ atoms in $\mathrm{Au} \mathrm{NCs}$, the intrinsic correlation between the ligand structure and catalytic performance, as well as the catalytic mechanism.

\subsection{What determines the stability of atomically precise $\mathrm{Au}$ nanoclusters?}

The premise for the application of Au NCs is to prepare stable ultrasmall $\mathrm{Au}$ NCs. According to the synthetic path, the reported preparation methods of Au NCs can be classified into "bottom-up" and "top-down" approaches. ${ }^{45}$ In the former, the precursor ions $\left(\mathrm{Au}^{3+}\right)$ are reduced to $\mathrm{Au}$ atoms $\left(\mathrm{Au}^{0}\right)$ under the action of reducing agent, and the resulting $\mathrm{Au}^{0}$ atoms are further combined with the ligands and then nucleated to grow into $\mathrm{Au}$ NCs. In the latter, the large $\mathrm{Au}$ NPs are etched by appropriate ligands to obtain ultrasmall NCs. From the viewpoint of reduction methods, the $\mathrm{Au} \mathrm{NC}$ preparation methods can be further divided into chemical reduction, ${ }^{46}$ photoreduction, ${ }^{47}$ ultrasonic reduction, ${ }^{48}$ microwave reduction, ${ }^{49}$ ligand exchange methods, ${ }^{50}$ etc. For details on the preparation methods of $\mathrm{Au}$ NCs, please refer to some excellent review articles. ${ }^{6,45,51,52}$

Regardless of the synthetic method, the reactants for the preparation of $\mathrm{Au}$ NCs usually contain ligand molecules in addition to the $\mathrm{Au}^{3+}$ precursor. During the reaction process, the ligand molecules combine with the $\mathrm{Au}^{3+}$ precursor ions, and eventually form the $\mathrm{Au}$ NCs consisting of the nucleus of $\mathrm{Au}^{0}$ atoms and the shell of the semi-ring $\mathrm{Au}(\mathrm{I}) /$ ligand staples or ligand molecules, as schematically illustrated in Fig. 2. For example, several groups ${ }^{53-55}$ confirmed the semi-ring $\mathrm{Au}(\mathrm{I}) /$ ligand staples on the surface of Au NCs by using advanced mass spectrometric techniques. In general, the stability of Au NCs depends on both the external environment (e.g., solution $\mathrm{pH}$ and temperature) and the internal structure, but the latter is fundamental for its stability. Therefore, the structural stability of Au NCs is mainly governed by the geometric structure of the $\mathrm{Au}$ atoms, the structure of the protecting ligand molecules, and the interaction between the two. The geometric structure of $\mathrm{Au}$ atoms determines the nucleus of NCs, ${ }^{56}$ while the ligand structure is closely related to the surface properties (e.g., hydrophilicity, ${ }^{57}$ polarity ${ }^{58}$ chirality, ${ }^{59}$ and functional modifiability ${ }^{60,61}$ ) of NCs, and the interaction between the two is reflected in the electronic and geometric properties of NCs. ${ }^{62}$

\section{What do we really know about the role of ligands in the nanoclusters' biocatalytic activities?}

\subsection{Origin of the biocatalytic activity of $\mathrm{Au}$ nanoclusters}

People have never stopped exploring the origin of the biocatalytic activities of Au NCs. In the early stage, it was generally 


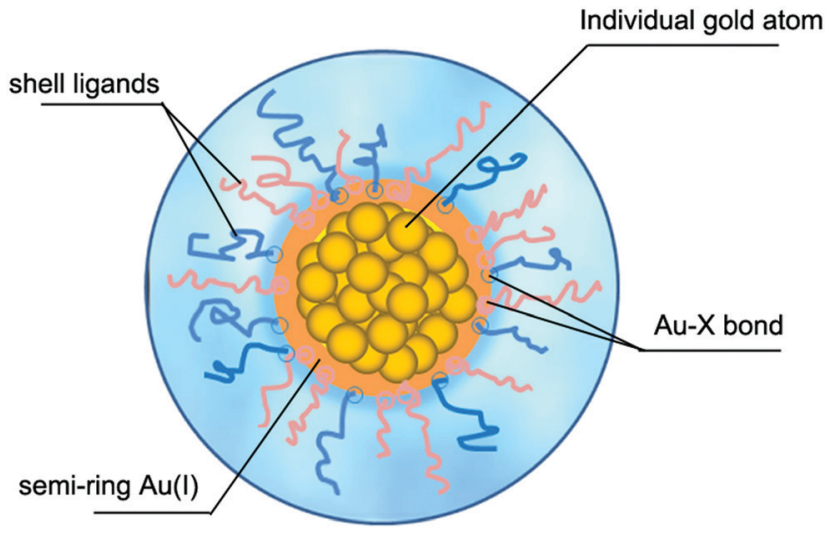

Au nanoclusters

Fig. 2 Schematic illustration of the structure of Au nanoclusters.

accepted that the biocatalytic activities of Au NCs were closely related to their fluorescence performances, which were mainly dependent on the $\mathrm{Au}$ atomic core instead of the ligand shell because the ligands only served to protect and stabilize the core. The most direct evidence is that the naked Au NCs, only composed of several Au atoms, have been confirmed to possess an obvious photoluminescence (PL) phenomenon, and the number of $\mathrm{Au}$ atoms also has an important influence on their PL performance. ${ }^{63,64}$ For example, Fedrigo et al. ${ }^{63}$ detected the PL properties of small naked Au NCs under inert atmosphere. Zheng et $a l^{65}$ experimentally revealed that the PL performance of Au NCs varied with the number of Au atoms, and the Jellium model $\left(E_{\mathrm{Fermi}} / N^{1 / 3}\right.$, where $E_{\mathrm{Fermi}}$ is the Fermi energy of bulk gold and $N$ is the number of Au atoms $)^{13}$ could on the whole reflect the internal correlation between the number of $\mathrm{Au}$ atoms and the PL of small sized Au NCs, although the model would be offset to some extent when the number of Au atoms was large. ${ }^{66}$ In addition, the PL performance of Au NCs was also demonstrated to change significantly when other metal atoms (e.g., $\mathrm{Ag}^{67}$ and $\mathrm{Cu}^{68}$ ) partially substituted the $\mathrm{Au}$ atoms in the core of Au NCs, implying that the PL of Au NCs originated from the core of Au NCs. For instance, Wang et al. ${ }^{67}$ revealed that the PL intensity of $\mathrm{Ag}_{x} \mathrm{Au}_{25-x}(x=13)$ NCs obtained by partially replacing $\mathrm{Au}$ atoms with $\mathrm{Ag}$ atoms was about 200 times that of $\mathrm{Au}_{25} \mathrm{NCs}$ (Fig. 3). Under the influence of these early viewpoints, the role of the ligand shell in the biocatalytic activities of $\mathrm{Au}$ NCs has been ignored for a long time.

With the development of research studies in recent years, it has been proven that the biocatalytic activities of Au NCs are not only determined by the Au atomic core, but also greatly influenced by the shell of the ligand molecules. For Au NCs with the same Au atomic core, the use of different shell ligands usually leads to the formation of Au NCs with isomerized structures, resulting in significantly different spectral absorption properties and ultimately affecting their catalytic activities, as exemplified in the work of Jin's group ${ }^{69}$ (Fig. 4). In some cases, the biocatalytic activities of Au NCs even come directly from the shell of the ligands. The influence of ligand shell on
A

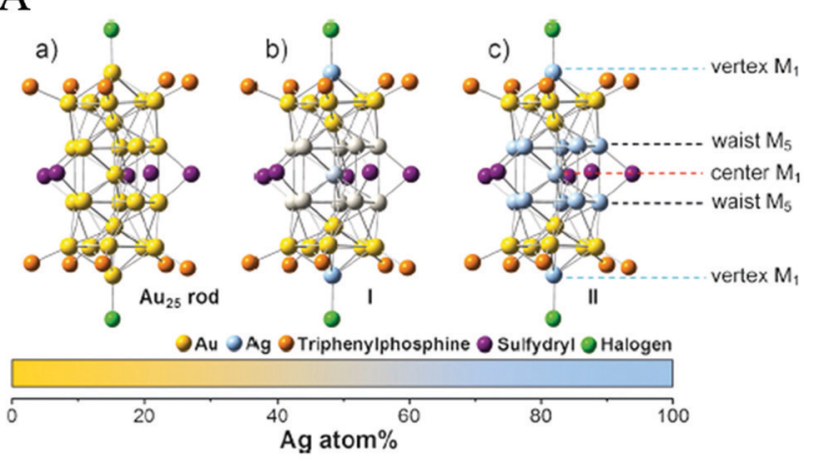

B

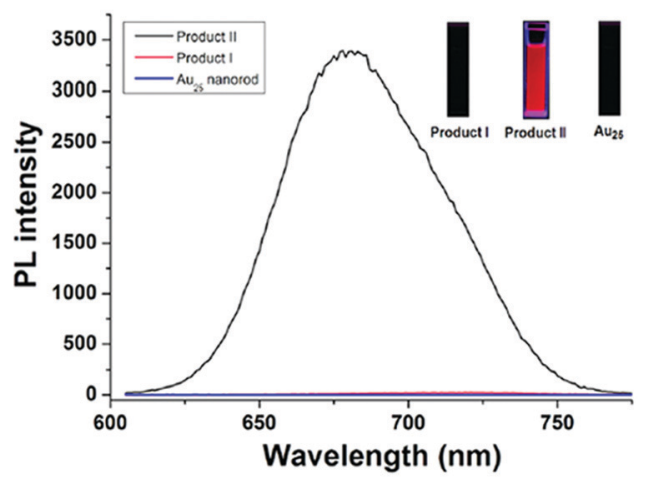

Fig. 3 (A) X-ray structures of: (a) $\mathrm{Au}_{25} \mathrm{NCs}$, (b) Product I ( $\mathrm{Ag}_{12} \mathrm{Au}_{13} \mathrm{NCs}$ ), and (c) Product II ( $\left.\mathrm{Ag}_{13} \mathrm{Au}_{12} \mathrm{NCs}\right) . \mathrm{M}=$ metal atom. (B) Photoluminescence spectra of $\mathrm{Au}_{25} \mathrm{NCs}$ (bottom, blue line), Product I $\left(\mathrm{Ag}_{12} \mathrm{Au}_{13} \mathrm{NCs}\right.$ ) (middle, red line) and Product II $\left(\mathrm{Ag}_{13} \mathrm{Au}_{12} \mathrm{NCs}\right)$ (top, black line), the inset is the fluorescence digital photo of $\mathrm{Au}_{25}$, Product I and Product II excited by $365 \mathrm{~nm}$ UV light, respectively. Reproduced from ref. 58 with permission. Copyright 2014 Wiley-VCH.

the biocatalytic activities of Au NCs is mainly reflected in the following aspects. Firstly, ligand molecules are bound to $\mathrm{Au}(\mathrm{I})$ via chemical bonds (e.g., Au-S) to form a semi-ring structure at the outer layer of the $\mathrm{Au}(0)$ core. The resulting semi-ring structure can, on the one hand, protect and stabilize the $\mathrm{Au}(0)$ core, and on the other hand also provide the charge transfer channel (i.e., the ligands to the metal cluster core charge transfer (LMCCCT)) for the biocatalytic process. The LMCCCT would effectively promote the interfacial charge transfer and separation, and thus change the fluorescence and biocatalytic performance of Au NCs. Link et al. ${ }^{70}$ for example, revealed that $\mathrm{Au}_{25}(\mathrm{SR})_{18}$ NCs exhibited two distinct characteristic fluorescence phenomena, in which the weak visible fluorescence emission (quantum yield $(\mathrm{QY})=\sim 10^{-6}-10^{-7}$ ) originated from the $\mathrm{Au}(0)$ kernel structure while the other strong near-infrared fluorescence emission $\left(\mathrm{QY}=\sim 1 \times 10^{-3}\right)$ originated from the semi-ring structure of the Au NCs. Interestingly, Miller et al. ${ }^{71}$ also observed two charge transfer processes with significantly different migration rates within the $\mathrm{Au} \mathrm{NCs}$, that is, the extremely fast charge transfer process $(<200 \mathrm{fs})$ in the $\mathrm{Au}(0)$ kernel and the relatively slow charge transport process $(\sim 1.2 \mathrm{ps})$ in the semi-ring structure, further revealing the important influence of the ligands on the fluorescence and biocatalytic reaction processes of Au NCs. Secondly, some ligands, as the shells of Au NCs, 

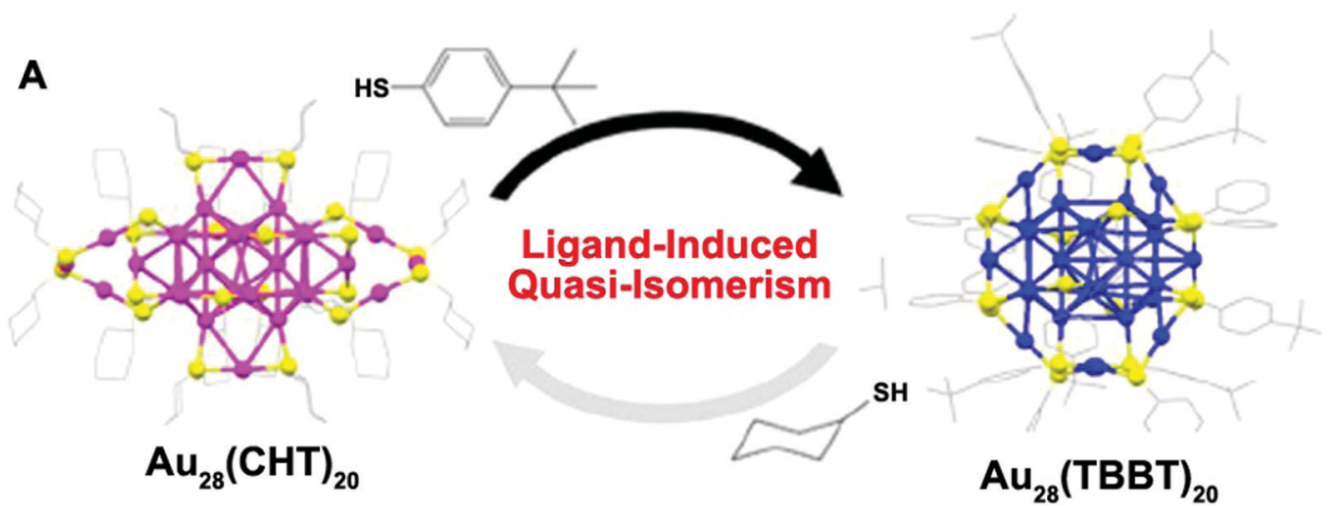

B

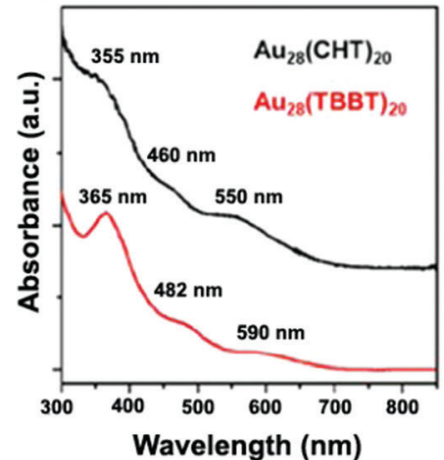

C

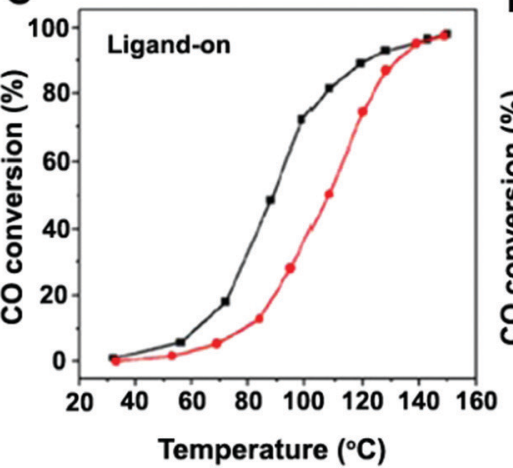

D

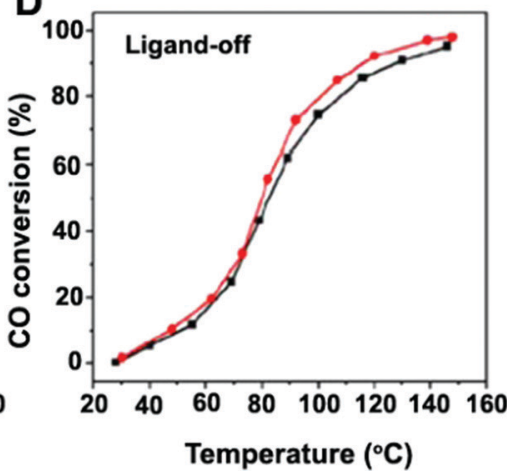

Fig. 4 (A) Ligand-induced quasi-isomerism in $\mathrm{Au}_{28}(\mathrm{CHT})_{20}$ and $\mathrm{Au}_{28}(\mathrm{TBBT})_{20}$, (CHT: cyclohexanethiolate; TBBT: 4-tert-butylbenzenethiolate); (B) optical absorption spectra of $\mathrm{Au}_{28}(\mathrm{CHT})_{20}$ (black) and $\mathrm{Au}_{28}(\mathrm{TBBT})_{20}$ (red). (C and D) $\mathrm{CO}$ oxidation light-off curves for $\mathrm{CeO}_{2}$-supported $\mathrm{Au}_{28}(\mathrm{CHT})_{20}$ (black) and $\mathrm{Au}_{28}(\mathrm{TBBT})_{20}$ (red) catalysts (C) pretreated with $\mathrm{O}_{2}$ at $150{ }^{\circ} \mathrm{C}$ for $1 \mathrm{~h}$ or (D) pretreated with $\mathrm{O}_{2}$ at $300{ }^{\circ} \mathrm{C}$ for $1 \mathrm{~h}$ to remove ligands. Reproduced from ref. 60 with permission. Copyright 2016 American Chemical Society.

are rich in donor electrons, which easily provides non-localized electrons that induce fluorescence and biocatalytic activities. ${ }^{72,73}$ For example, glutathione-protected Au NCs exhibited excellent fluorescence performance, mainly because of the presence of electron-rich atoms (e.g., $\mathrm{O}, \mathrm{N})$ or atom groups $(e . g .,-\mathrm{COOH}$, $-\mathrm{NH}_{2}$ ) in the glutathione ligands that greatly promoted the LMCCCT process. ${ }^{73}$ Thirdly, some ligands, especially some proteins, used as shells have their own biocatalytic activities, while stabilizing and protecting the $\mathrm{Au}(0)$ core $^{74-77}$ For example, $\mathrm{Au}$ NCs prepared with histidine as shell ligands showed peroxidase-like catalytic activity, ${ }^{75}$ while the introduction of bilirubin oxidase (BOD) into Au NCs as the shell ligands endowed the $\mathrm{Au}$ NCs with the catalytic activity for the oxygen reduction reaction (ORR). ${ }^{76}$ Lastly, the shell ligands of Au NCs can be further coupled with other functional molecules with biocatalytic activity (e.g., fungicides, ${ }^{78}$ folic acid, ${ }^{79,80}$ and anticancer drugs ${ }^{81}$ ) to form functionally-modified Au NCs, thus enabling the biocatalytic activities of Au NCs. Based on the preparation of DT-Au NCs with 1-dodecanethiol (DT) as the shell, for instance, Chen et $a .^{78}$ further introduced a bactericidal polypeptide molecule (i.e., surfactin (SFT)) on the surface of DT-Au NCs and thus prepared SFT/DT-Au NCs. The obtained SFT/DT-Au NCs possessed excellent biocompatibility and bactericidal properties, enabling the rapid healing of the methicillin-resistant $S$. aureus infected wounds in mice.

\subsection{Ligand species reported so far}

The utilization of the capping/stabilizing ligands has been demonstrated to reduce and arrange gold atoms into stable $\mathrm{Au}$ NCs. In order to obtain stable and monodisperse Au NCs, the shell ligands are indispensable and must also meet some requirements: (i) good structural stability, (ii) a relatively strong binding force with $\mathrm{Au}$ atoms, (iii) good solubility, and (iv) confined space. To date, most of the reported shell ligands are bound with the $\mathrm{Au}$ atoms through strong $\mathrm{Au}-\mathrm{S}$ bonds to form $\mathrm{Au} \mathrm{NCs},{ }^{82-84}$ while the other few ligands are bound with the $\mathrm{Au}$ atoms through weak bonds (such as phosphine, ${ }^{85}$ alkynyl $^{86}$ ). Apparently, the weakly-bonded shell ligands of the Au-NCs have poor stability, and some of them may detach from the surface of Au atoms in solution, thus leading to the formation of "semi-naked" or "fully naked" Au atomic clusters. These naked Au atomic clusters without the protective layer are easy to agglomerate and no longer have the structural characteristics of $\mathrm{Au}$ NCs. For some catalytic reactions, however, the naked $\mathrm{Au}$ atomic clusters can instead provide more active sites, which would be conducive to the catalytic processes. ${ }^{6}$

Currently, there are many kinds of capping/protecting ligands used for the preparation of Au NCs, mainly including small organic molecules (e.g., thiolate, ${ }^{52,87}$ phosphine ${ }^{85,88}$ ), dendrimer or polymer, ${ }^{89}$ biological molecules (such as peptides, ${ }^{90}$ protein, $\left.{ }^{19} \mathrm{DNA}^{76,91}\right)$, etc. In comparison, when used 
as shell ligands, small organic molecules are more competitive for the design and synthesis of Au NCs, because the structure of $\mathrm{Au}$ NCs can be easily regulated by changing the chain length, polarity or functional groups of the small organic molecules. For example, when alkanethiols are used as shell ligands, changing their chain length can produce Au NCs with different structures, thus resulting in different fluorescence effects ${ }^{92}$ (Fig. 5). When using dendrimers or polymers as shell ligands, it is difficult to regulate the structure of Au NCs because of their large and complex molecular structure. The dendrimer or polymer shell generally occupies much more space than the space occupied by the $\mathrm{Au}(0)$ core, resulting in poor monodispersity of $\mathrm{Au}$ NCs, and meanwhile the number of $\mathrm{Au}$ atoms in the $\mathrm{Au}$ NCs is not easy to determine. ${ }^{6}$ Consequently there are few reports on this type of Au NC. Since the first report on the preparation of $\mathrm{Au}$ NCs obtained by using bovine serum albumin (BSA) as the shell ligands, ${ }^{18}$ many biomolecules have been used as shell ligands for the preparation of $\mathrm{Au}$ NCs, including proteins (e.g., BSA, ${ }^{19,93}$ lysozyme,${ }^{94}$ human transferrin, ${ }^{95}$ lactoferrin, ${ }^{96}$ trypsin, ${ }^{97}$ horseradish peroxidase ${ }^{98}$ ), peptides, ${ }^{90,99,100}$ DNA, ${ }^{76,101}$ etc. The Au NCs protected by the bio-ligands have several unique advantages: (i) the improved biocompatibility of $\mathrm{Au}$ NCs, (ii) the introduction of surface functional groups (e.g., $\left.-\mathrm{NH}_{2},-\mathrm{COOH}\right)$ into the $\mathrm{Au} \mathrm{NCs}$, and (iii) the possible biocatalytic activities originated from some shell bio-ligands (e.g., enzyme $\left.{ }^{98}\right)$. These advantages can facilitate the applications of Au NCs in bioanalytical and biomedical fields.

\subsection{Ligand's role in the nanoclusters' biocatalytic activities}

Ligands are an indispensable part of Au NCs and play an important role in the biocatalytic activities of Au NCs. In the process of biocatalytic reaction, ligands, on one hand, are the stable and protective layers of Au NCs, and on the other hand, are the link between Au NCs and biological media for mass migration and charge transfer, which largely determines the biocompatibility and biocatalytic activities of Au NCs. Now the question is, how much do we know about the roles of the ligands?

3.3.1 What is known? Firstly, the shell ligands are the biocatalytic reaction interface of $\mathrm{Au}$ NCs, because the shell ligands are in direct contact between the Au NCs and environmental media. Generally speaking, the biocatalytic reaction occurs in solution, while the processes of mass migration and charge transfer between Au NCs dispersed in solution and the biological media are accomplished through the shell ligands. Meanwhile, the dispersity of Au NCs also depends on their shell ligands. The better the dispersity of the Au NCs, the more the shell ligands exposed to solution will be, which means that the more biocatalytic reaction interface provided by $\mathrm{Au}$ NCs, the more favorable the biocatalytic reaction will be.

Secondly, shell ligands provide reactive sites for the biocatalytic process of $\mathrm{Au}$ NCs, because shell ligands usually
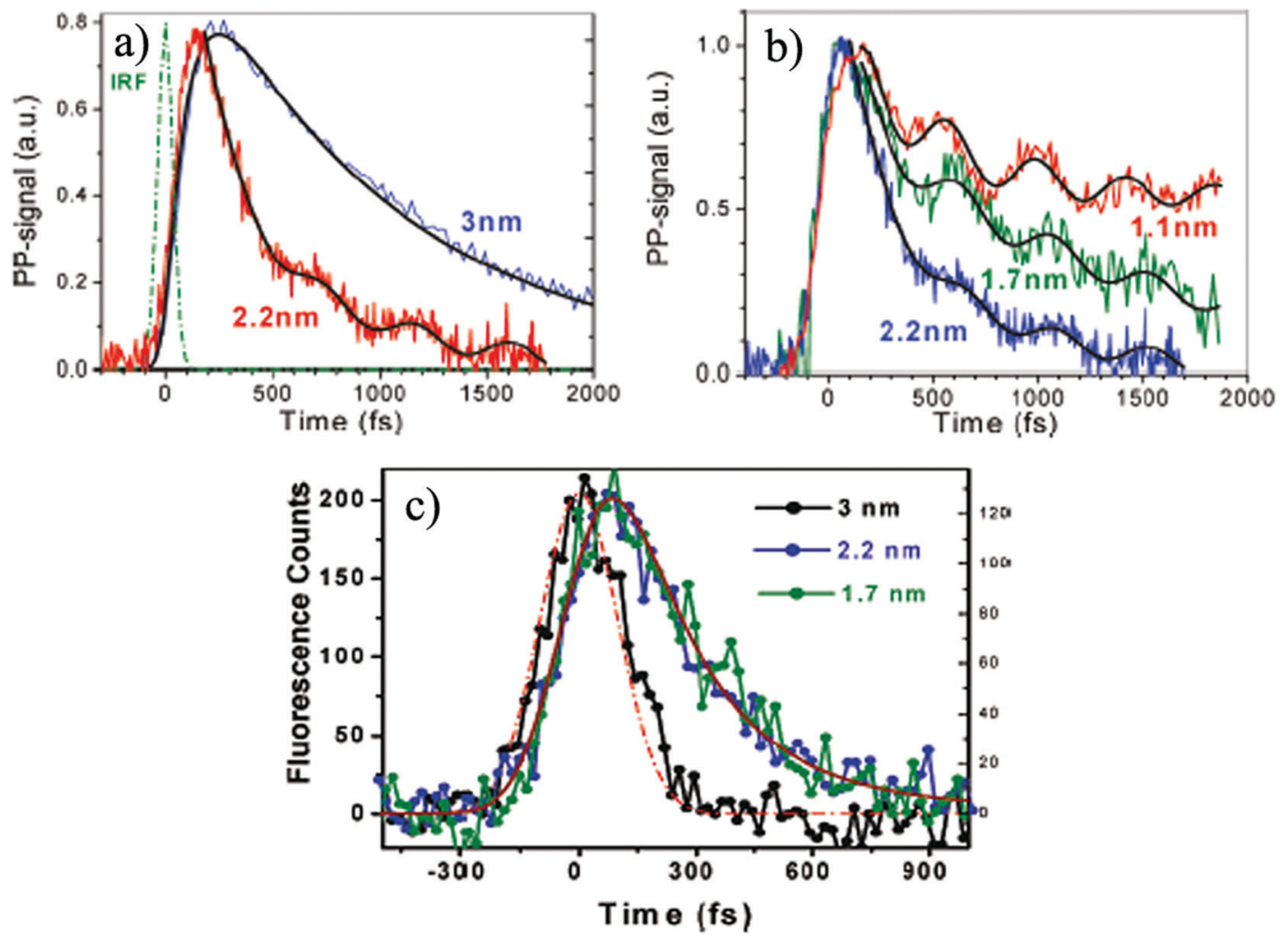

Fig. 5 (a) Demonstration of different transient absorption dynamics for Au NCs with the cluster sizes of 2.2 and 3 nm; (b) decay profiles with oscillatory features for Au NCs with the cluster sizes of 1.1, 1.7 and $2.2 \mathrm{~nm}$; (c) time-resolved fluorescence profiles for different Au NCs with the cluster sizes of 1.7, 2.2 and $3 \mathrm{~nm}$ measured at $530 \mathrm{~nm}$. Reproduced from ref. 83 with permission. Copyright 2010 American Chemical Society. 
contain many functional groups that can participate in the biocatalytic process. When a biocatalytic process occurs, $\mathrm{Au}$ NCs can adsorb and interact with the biological analytes/ objects in the solution through some functional groups existing on the surface of the shell ligands, and the charge transfer process between Au NCs and the biological analytes/objects is also realized. The more reactive sites provided by ligands, the higher the biocatalytic reactivity of Au NCs is.

Thirdly, shell ligands can be used to regulate the biocatalytic selectivity of Au NCs, due to the structural modulability of shell ligands. For a specific biocatalytic reaction process, specific reaction sites can be introduced into ligands by selecting appropriate shell ligands or modifying ligands with functional groups. Accordingly, it can induce a specific biocatalytic reaction process and greatly improve the biocatalytic selectivity of Au NCs. For example, the introduction of antibodies on the shell ligand surface of the Au NCs would lead to a specific biological reaction with the receptor in the biological medium, showing excellent biocatalytic selectivity. ${ }^{102-104}$

In addition, shell ligands can improve the biocompatibility of Au NCs in biocatalytic reactions. When a biocatalytic process occurs, $\mathrm{Au}$ NCs are bound to interact with various biological molecules, cells or tissues. Therefore, the biocompatibility of $\mathrm{Au}$ NCs is crucial. In recent years, the use of various biological molecules (e.g., proteins, peptides, DNA, as mentioned above) as the shell ligands of Au NCs has greatly improved the biocompatibility of Au NCs and further reduced the biological toxicity. ${ }^{101}$

3.3.2 What is not known? Although Au NCs show excellent biocatalytic activities and in recent years have been widely used for bioanalytical and biomedical applications, the biocatalytic mechanism of $\mathrm{Au}$ NCs is not clear, and there are many problems that remain to be further explored. In particular, the exploration on the effect of the shell ligands on the biocatalytic activities of Au NCs is one of the problems that needs to be addressed.

Shell ligands have diverse structural features. When a biocatalytic reaction occurs, the shell ligands of Au NCs interact with environmental media, resulting in the diversification of shell ligands. The diversity of shell ligands not only depends on their own molecular structures, but also is closely related to the environmental media (e.g., spatial charge distribution of shell ligands, polarity or solubility of ligands, and spatial distance of adjacent $\mathrm{Au}$ NCs). However, whether their own molecular structures or external factors determine the diversity characteristics of shell ligands has not been determined yet, and further research is needed.

As is well-known, Au NCs, different from the metallic Au NPs, have a molecular-like electronic structure, that is, the HOMO-LUMO structure, ${ }^{8,9}$ which is an essential intrinsic factor in determining their charge transfer behavior in the biocatalytic reaction. However, it is not clear how shell ligands participate in the formation of the HOMO-LUMO electronic structure of $\mathrm{Au}$ NCs and how they affect the electronic structure. Moreover, the influence of shell ligands on the charge transfer behavior of Au NCs during the biocatalytic reaction processes is also a blind spot, which needs to be further explored. According to Marcus Theory ${ }^{105}$ formula:

$$
k_{\mathrm{ET}}=A_{\mathrm{ET}} \mathrm{e}^{-\Delta G / R T}
$$

where $k_{\mathrm{ET}}$ is the electron transfer rate, $A_{\mathrm{ET}}$ is the rate constant, $\Delta G$ is free energy changes, $R$ is the ideal gas constant, and $T$ is the absolute temperature. $A_{\mathrm{ET}}$ declines exponentially with the distance between the donor and receptor, and thus $k_{\mathrm{ET}}$ decreases exponentially. This indicates that the size of the shell ligands significantly affects the charge transfer rate of $\mathrm{Au}$ NCs. Theoretically, the larger the shell ligands, the smaller the charge transfer rate of Au NCs. However, when a biocatalytic reaction process occurs, the charge transfer rate of Au NCs is not only affected by the size of the ligands, but also by their surface charge. Moreover, the shell ligands are always in a dynamic process, which also affects the charge transfer process of Au NCs. Therefore, from the perspective of the reaction mechanism, it is still unknown what effect the shell ligands have on the charge transfer behavior of $\mathrm{Au}$ NCs and their biocatalytic reaction kinetics.

As mentioned above, when a biocatalytic reaction occurs, Au NCs usually adsorb biological analytes/objects through the shell ligands and interact with them, and at the same time the charge transfer behavior is also generated between the two. Apparently, shell ligands are the link between Au NCs and the bioanalyte/object, and also the bridge of charge transfer between the two in the biocatalytic reaction processes. Studies have shown that the charge transfer at the interface between $\mathrm{Au}$ NCs usually involves electron hopping ${ }^{106,107}$ or quantum tunneling behavior. ${ }^{108,109}$ However, the role of the shell ligands in the charge transfer process (e.g., electron hopping or quantum tunneling) between $\mathrm{Au}$ NCs and bioanalytes/objects is still unknown, and the impact of shell ligands on the charge transfer mechanism of $\mathrm{Au}$ NCs is also unclear. In other words, shell ligands can generally induce the surface charge redistribution of Au NCs. What needs to be clarified is the difference between the charge distribution induced by ligands and the charge transfer mechanism of Au NCs.

The spatial structure of the shell ligands determines the actual particle size of Au NCs and affects the biocatalytic activities of Au NCs. Though the reported synthesis processes can control the particle size of Au NCs to some extent, they cannot accurately regulate the amount and surface charge of shell ligands on the surface of $\mathrm{Au}$ NCs, resulting in poor reproducibility of Au NCs. As a result, the spatial structure of shell ligands for different $\mathrm{Au}$ NCs is diverse, thus affecting the biocatalytic activities of Au NCs. Therefore, it is a great challenge for $\mathrm{Au}$ NCs to precisely regulate the spatial structure of shell ligands through the synthetic process, so as to control the structure and biocatalytic activities of Au NCs. Undoubtedly, this is also one of the urgent problems to be solved.

The biocatalytic activities of Au NCs are closely related to the microscopic states (e.g., the distribution density, the uniformity, and the surface charge) of shell ligands on the surface of Au NCs. However, the existing characterization equipment and techniques are still unable to detect the microscopic states 
of the shell ligands of Au NCs, resulting in our limited understanding on the microscopic states of shell ligands. Therefore, currently it is still difficult to establish the intrinsic correlation between shell ligands and the biocatalytic activities of Au NCs. Nevertheless, this is a problem that must be solved to explore the influence of ligands on the biocatalytic activities of Au NCs.

\section{Summary and future prospects}

Au nanoclusters (NCs), due to their unique electronic structure, excellent fluorescence performance, good biocompatibility and ability to be removed by renal clearance, have shown more excellent biocatalytic activities than Au NPs. In recent years, Au NCs have attracted extensive attention and have been widely applied in the field of biological analysis and biomedicine. As an important part of Au NCs, shell ligands not only stabilize and protect the structure of Au NCs, but also have important effects on the structure and performance of $\mathrm{Au}$ NCs. In the process of biocatalytic reaction, obviously, shell ligands also affect the biocatalytic activity of $\mathrm{Au}$ NCs to a great extent. At present, however, the effect of ligands on the biocatalytic activities of Au NCs has not been paid much attention or even ignored. In this Focus article, from the perspective of ligands, the effect of ligands on the biocatalytic activity of Au NCs is briefly analyzed, and the important role of ligands in the biocatalytic reaction of Au NCs is also discussed. Meanwhile, some unknown problems to be further explored are put forward.

The importance of shell ligands on the structure and biocatalytic activity of Au NCs is self-evident, and thus it is of great significance to reveal the role of shell ligands in the biocatalytic activity of Au NCs. Nevertheless, research toward the effect of shell ligands on the structure and biocatalytic activities of $\mathrm{Au}$ NCs remains extremely challenging. Much work remains to be done in facilitating their practical applications of Au NCs and broadening the scope of their bio-related applications in the future. Specific attention should be focused on a range of current challenges, such as facile synthesis and functionalization of stable and monodisperse ligand-protected Au NCs, the determination of the structure of Au NCs at the atomic level, further exploration of the electronic structure of $\mathrm{Au}$ NCs, a better understanding of the effect of shell ligands on the charge transfer behavior of $\mathrm{Au} \mathrm{NCs,} \mathrm{and} \mathrm{the} \mathrm{interpretation} \mathrm{of} \mathrm{the}$ charge transport characteristics at the interface between the shell ligands and the $\mathrm{Au}(0)$ core of $\mathrm{Au} \mathrm{NCs}$, or the interface between the shell ligands and the bioanalytes in solution. In view of this, we believe that the following aspects are the future directions to explore the mechanism of shell ligands affecting the biocatalytic activities of Au NCs, as schematically presented in Fig. 6.

\section{(1) Surface ligand engineering of Au NCs}

The current synthetic processes of Au NCs seem to be able to effectively regulate the particle size of NCs to a certain extent, but it is far from enough to just regulate the particle size of NCs. This is because the inability to design and regulate the

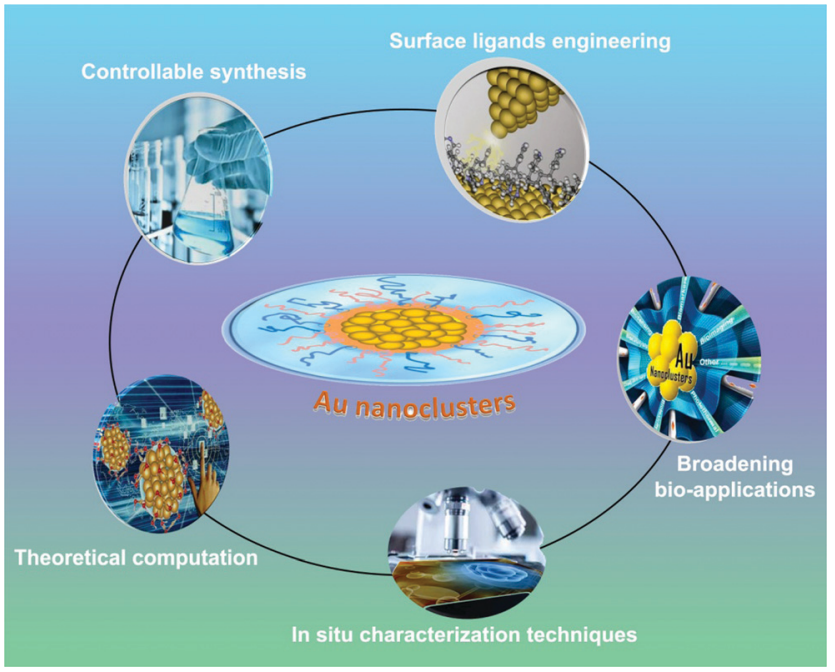

Fig. 6 Schematic illustration of the future directions to explore the mechanisms of shell ligands affecting the biocatalytic activities of $\mathrm{Au}$ nanoclusters.

ligands on the Au NCs' surface makes it impossible to achieve the reproducibility of Au NCs' synthesis and even less to regulate their biocatalytic activities, which highlights the importance of surface ligand engineering of Au NCs. The most important content of surface ligand engineering of Au NCs is how to precisely regulate the shell ligands of Au NCs through new synthetic methodologies. The precise regulation of shell ligands is a prerequisite for the controllable synthesis of fine-structure $\mathrm{Au}$ NCs, and is also an indispensable condition for subsequent research on the influence mechanism of shell ligands on the biocatalytic activities of $\mathrm{Au}$ NCs. In order to realize the precise regulation of ligands on the surface of $\mathrm{Au}$ NCs, it is necessary to explore the distribution uniformity, the binding stability, the surface charge state and the threedimensional spatial structure of ligands, etc., on the surface of $\mathrm{Au}$ NCs. All of these belong to the research category of surface ligand engineering of $\mathrm{Au}$ NCs.

\section{(2) Development of advanced in situ characterization techniques for surface ligands}

In order to understand the mechanism of ligands' influence on the biocatalytic activities of $\mathrm{Au}$ NCs, it is necessary to in situ analyze the microstructure and charge distribution of shell ligands. However, the currently-reported nanoscale surface/ interface characterization techniques (such as high-resolution transmission electron microscopy (HRTEM), atomic force microscopy (AFM), X-ray photoelectron spectroscopy (XPS), scanning tunneling microscopy (STM), and Kelvin probe microscopy (KPM)) are commonly used to examine the microstructure and morphologies of the NPs themselves, rarely involving the in situ detection of the morphology and charge distribution of ligands on the surface of NPs. Moreover, the shell ligands change dynamically during the biocatalytic reaction of Au NCs, and there are few characterization techniques for the in situ monitoring of the dynamic changes of ligands in real time. It is worth 
mentioning that single-atom catalysis has become a research hotspot in the field of catalysis in recent years, while the characterization techniques on single-atom catalysts (such as high-angle annular dark field-scanning transmission electron microscopy (HAADF-STEM), X-ray absorption near edge spectroscopy (XANES), and extended X-ray absorption fine structure (EXAFS) spectroscopy) lay more emphasis on the single-atom catalysts themselves than on their surface ligands. ${ }^{110}$ Therefore, the development of advanced nano-surface/interface in-situ characterization techniques (e.g., surface enhanced Raman spectroscopy (SERS), diffuse reflectance infrared Fourier transform (DRIFT-IR), electrospray ionization mass spectrometry (ESI-MS), matrix-assisted laser desorption/ionization-mass spectrometry (MALDI-TOF-MS), XANES, EXAFS, and surface probes) is very important for the in situ characterizations on the microstructure and charge distribution of shell ligands of Au NCs in biocatalytic reactions. Meanwhile, it is also helpful to reveal the mechanism of shell ligands' influence on the biocatalytic activities of Au NCs.

\section{(3) Theoretical analysis of the shell ligands of Au NCs}

At present, there are few theoretical studies on the effect of shell ligands on the charge transfer behavior, biocatalytic activities and reaction kinetics of Au NCs. Therefore, on the basis of the building of a computational model for different ligand-based Au NCs, the theoretical exploration of the intrinsic correlation between the shell ligands and the charge transfer behavior as well as biocatalytic kinetics of Au NCs will help to deepen the understanding of the role of shell ligands in the biocatalytic process of Au NCs, which is of great significance to reveal the biocatalytic mechanism of Au NCs.

\section{(4) Broadening the nanobiological applications of Au NCs}

As mentioned above, when a biocatalytic reaction of Au NCs occurs, the shell ligands are in direct contact with the environmental media, which are usually complicated for any biological system. In some biological systems, the interaction of the shell ligands with the environmental media may induce the aggregation of Au NCs, thus leading to the loss of their original physical structures. In other biological systems, the interaction of ligands with the environmental media may contribute to the dispersion and stabilization of Au NCs and thus promote the biocatalytic process. In addition, when Au NCs in the original biological system enter into another new biological system, the interaction of shell ligands with the environmental media also changes, which may promote or inhibit the biocatalytic activities of Au NCs. In other words, the shell ligands of Au NCs largely determine the biological system applicable to Au NCs and even the extended application of Au NCs in other fields. Thus, the clear understanding of the relationship between shell ligands and the biocatalytic activities of $\mathrm{Au}$ NCs is of great significance to broaden the nanobiological applications of $\mathrm{Au}$ NCs, such as sensing analysis, nanocatalysis, drug therapy and biological toxicity.

Despite these remaining challenges, it is clear that $\mathrm{Au}$ NCs offer many advantages for a wide range of bio-related applications, and currently researchers are making rapid progress in this area.

\section{Conflicts of interest}

There are no conflicts to declare.

\section{Acknowledgements}

This work is financially supported by the National Natural Science Foundation of China (No. 51972294, 21621003, 51872271), National Key Research and Development Program of China (No. 2016YFA0203101), Zhejiang Provincial Natural Science Foundation of China (No. LY19E020003, LQ20F040007), and Tsinghua University Initiative Scientific Research Program.

\section{References}

1 L. Y. Chen, C. W. Wang, Z. Q. Yuan and H. T. Chang, Fluorescent Gold Nanoclusters: Recent Advances in Sensing and Imaging, Anal. Chem., 2015, 87, 216-229.

2 R. C. Jin, C. J. Zeng, M. Zhou and Y. X. Chen, Atomically Precise Colloidal Metal Nanoclusters and Nanoparticles: Fundamentals and Opportunities, Chem. Rev., 2016, 116, 10346-10413.

3 Y. Z. Lu and W. Chen, Sub-nanometre sized metal clusters: from synthetic challenges to the unique property discoveries, Chem. Soc. Rev., 2012, 41, 3594-3623.

4 I. Díez and R. Ras, Advanced Fluorescence Reporters in Chemistryand Biology II, Springer, 2010, pp. 307-332.

5 L. B. Zhang and E. K. Wang, Metal nanoclusters: New fluorescent probes for sensors and bioimaging, Nano Today, 2014, 9, 132-157.

6 J. Fang, B. Zhang, Q. F. Yao, Y. Yang, J. P. Xie and N. Yan, Recent advances in the synthesis and catalytic applications of ligand protected,atomically precise metal nanoclusters, Coord. Chem. Rev., 2016, 322, 1-29.

7 Y. Zhao, Y. Q. Zheng, C. Y. Zhao, J. M. You and F. L. Qu, Hollow PDA-Au nanoparticles-enabled signal amplification for sensitive nonenzymatic colorimetric immune detection of carbohydrate antigen125, Biosens. Bioelectron., 2015, 71, 200-206.

8 A. Fernando, K. L. D. M. Weerawardene, N. V. Karimova and C. M. Aikens, Quantum Mechanical Studies of Large Metal, Metal Oxide, and Metal Chalcogenide Nanoparticles and Clusters, Chem. Rev., 2015, 115, 6112-6216.

9 M. Z. Zhu, C. M. Aikens, F. J. Hollander, G. C. Schatz and R. C. Jin, Correlating the Crystal Structure of A ThiolProtected $\mathrm{Au}_{25}$ Cluster and Optical Properties, J. Am. Chem. Soc., 2008, 130, 5883-5885.

10 S. W. Chen, R. S. Ingram, M. J. Hostetler, J. J. Pietron, R. W. Murray, T. G. Schaaff, J. T. Khoury, M. M. Alvarez and R. L. Whetten, Gold Nanoelectrodes of Varied Size: Transition to Molecule-Like Charging, Science, 1998, 280, 2098-2101. 
11 S. Knoppe and T. Bürgi, Chirality in Thiolate-Protected Gold Clusters, Acc. Chem. Res., 2014, 47, 1318-1326.

12 L. Shang, S. Dong and G. U. Nienhaus, Ultra-small fluorescent metal nanoclusters: Synthesis and biological applications, Nano Today, 2011, 6, 401-418.

13 N. Goswami, Q. F. Yao, Z. T. Luo, J. G. Li, T. K. Chen and J. P. Xie, Luminescent Metal Nanoclusters with AggregationInduced Emission, J. Phys. Chem. Lett., 2016, 7(6), 962-975.

14 G. Li and R. C. Jin, Atomically Precise Gold Nanoclusters as New Model Catalysts, Acc. Chem. Res., 2013, 46, 1749-1758.

15 W. Kurashige, Y. Niihori, S. Sharma and Y. Negishi, Precise synthesis, functionalization and application of thiolateprotected gold clusters, Coord. Chem. Rev., 2016, 320-321, 238-250.

16 Y. Liu, X. L. Li, H. Liu, Z. J. Zhou, J. P. Huang, S. L. Lei, S. H. Cai, Z. Chen, Y. L. Guo, Z. W. Chen, X. Zhou and L. M. Nie, Porous gold nanocluster-decorated manganese monoxide nanocomposites for microenvironment-activatable MR/photoacoustic/CT tumor imaging, Nanoscale, 2018, 10, 3631-3638.

17 G. Hughes and J. C. Lewis, Introduction: Biocatalysis in Industry, Chem. Rev., 2018, 118, 1-3.

18 R. H. Terrill, T. A. Postlethwaite, C. Chen, C. D. Poon, A. Terzis, A. Chen, J. E. Hutchison, M. R. Clark, G. Wignall, J. D. Londono, R. Superfine, M. Falvo, C. S. Johnson Jr., E. T. Samulski and R. W. Murray, Monolayers in Three Dimensions: NMR, SAXS, Thermal, and Electron Hopping Studies of Alkanethiol Stabilized Gold Clusters, J. Am. Chem. Soc., 1995, 117, 12537-12548.

19 J. P. Xie, Y. G. Zheng and J. Y. Ying, Protein-Directed Synthesis of Highly Fluorescent Gold Nanoclusters, J. Am. Chem. Soc., 2009, 131, 888-889.

20 M. S. Devadast, J. Kim, E. Sinn, D. Lee, T. Goodson and G. Ramakrishna, Unique Ultrafast Visible Luminescence in Monolayer-Protected $\mathrm{Au}_{25}$ Clusters, J. Phys. Chem. C, 2010, 114, 22417-22423.

21 J. Sun and Y. D. Jin, Fluorescent $\mathrm{Au}$ nanoclusters: recent progress and sensing applications, J. Mater. Chem. C, 2014, 2, 8000-8011.

22 Y. K. Zheng, W. W. Liu, Y. Chen, H. Jiang and X. M. Wang, Mercaptopyrimidine-directed gold nanoclusters: a suitable fluorescent probe for intracellular glutathione imaging and selective cancer cell identification, J. Mater. Chem. B, 2018, 6, 3650-3654.

23 X. Wen, P. Yu, Y. R. Toh and J. Tang, Structure-correlated dual fluorescent bands in BSA-protected $\mathrm{Au}_{25}$ nanoclusters, J. Phys. Chem. C, 2012, 116, 11830-11836.

24 K. R. Krishnadas, A. Ghosh, A. Baksi, I. Chakraborty, G. Natarajan and T. Pradeep, Intercluster Reactions between $\mathrm{Au}_{25}(\mathrm{SR})_{18}$ and $\mathrm{Ag}_{44}(\mathrm{SR})_{30}$, J. Am. Chem. Soc., 2016, 138, 140-148.

25 Q. Li, A. Das, S. X. Wang, Y. X. Chen and R. C. Jin, Highly efficient three-component coupling reaction catalysed by atomically precise ligand-protected $\mathrm{Au}_{38}\left(\mathrm{SC}_{2} \mathrm{H}_{4} \mathrm{Ph}\right)_{24}$ nanoclusters, Chem. Commun., 2016, 52, 14298-14301.

26 Y. Tao, Y. H. Lin, Z. Z. Huang, J. S. Ren and X. G. Qu, Incorporating Graphene Oxide and Gold Nanoclusters:
A Synergistic Catalyst with Surprisingly High PeroxidaseLike Activity Over a Broad pH Range and its Application for Cancer Cell Detection, Adv. Mater., 2013, 25, 2594-2599.

27 D. H. Hu, Z. H. Sheng, S. T. Fang, Y. N. Wang, D. Y. Gao, P. F. Zhang, P. Gong, Y. F. Ma and L. T. Cai, Folate Receptor-Targeting Gold Nanoclusters as Fluorescence Enzyme Mimetic Nanoprobes for Tumor Molecular Colocalization Diagnosis, Theranostics, 2014, 4, 142-153.

28 H. D. Cui, D. H. Hu, J. N. Zhang, G. H. Gao, Z. Chen, W. J. Li, P. Gong, Z. H. Sheng and L. T. Cai, Gold Nanoclusters-Indocyanine Green Nanoprobes for Synchronous Cancer Imaging, Treatment, and Real-Time Monitoring Based on Fluorescence Resonance Energy Transfer, ACS Appl. Mater. Interfaces, 2017, 9, 25114-25127.

29 X. Yu, C. X. Zhang, L. N. Zhang, Y. R. Xue, H. W. Li and Y. Q. Wu, The construction of a FRET assembly by using gold nanoclusters and carbon dots and their application as a ratiometric probe for cysteine detection, Sens. Actuators, B, 2018, 263, 327-335.

30 J. J. Yang, F. L. Wang, H. Q. Yuan, L. S. Zhang, Y. Y. Jiang, X. Zhang, C. Liu, L. Chai, H. Li and M. Stenzel, Recent advances in ultra-small fluorescent $\mathrm{Au}$ nanoclusters toward oncological research, Nanoscale, 2019, 11, 17967-17980.

31 Q. Tang, G. X. Hu, V. Fung and D. E. Jiang, Insights into Interfaces, Stability, Electronic Properties, and Catalytic Activities of Atomically Precise Metal Nanoclusters from First Principles, Acc. Chem. Res., 2018, 51, 2793-2802.

32 J. Z. Yan, B. K. Teo and N. F. Zheng, Surface Chemistry of Atomically Precise Coinage-Metal Nanoclusters: From Structural Control to Surface Reactivity and Catalysis, Acc. Chem. Res., 2018, 51, 3084-3093.

33 Y. Wang, X. K. Wan, L. T. Ren, H. F. Su, G. Li, S. Malola, S. C. Lin, Z. C. Tang, H. Häkkinen, B. K. Teo, Q. M. Wang and N. F. Zheng, Atomically Precise Alkynyl-Protected Metal Nanoclusters as a Model Catalyst: Observation of Promoting Effect of Surface Ligands on Catalysis by Metal Nanoparticles, J. Am. Chem. Soc., 2016, 138, 3278-3281.

34 X. K. Wan, J. Q. Wang, Z. A. Nan and Q. M. Wang, Ligand effects in catalysis by atomically precise gold nanoclusters, Sci. Adv., 2017, 3, e1701823.

35 Z. M. Li, C. Liu, H. Abroshan, D. R. Kauffman and G. Li, Au38S2(SAdm)20 Photocatalyst for One-Step Selective Aerobic Oxidations, ACS Catal., 2017, 7, 3368-3374.

36 X. F. Cui, J. Wang, B. Liu, S. Ling, R. Long and Y. J. Xiong, Turning Au Nanoclusters Catalytically Active for VisibleLight-Driven $\mathrm{CO}_{2}$ Reduction through Bridging Ligands, J. Am. Chem. Soc., 2018, 140, 16514-16520.

37 M. R. Narouz, K. M. Osten, P. J. Unsworth, R. W. Y. Man, K. Salorinne, S. Takano, R. Tomihara, S. Kaappa, S. Malola, C. T. Dinh, J. D. Padmos, K. Ayoo, P. J. Garrett, M. Nambo, J. H. Horton, E. H. Sargent, H. Häkkinen, T. Tsukuda and C. M. Crudden, N-heterocyclic carbene-functionalized magic-number gold nanoclusters, Nat. Chem., 2019, 11, 419-425.

38 S. Zhao, N. Austin, M. Li, Y. B. Song, S. D. House, S. Bernhard, J. C. Yang, G. Mpourmpakis and R. C. Jin, 
Influence of Atomic-Level Morphology on Catalysis: The Case of Sphere and Rod-Like Gold Nanoclusters for CO2 Electroreduction, ACS Catal., 2018, 8, 4996-5001.

39 G. X. Zhao, H. M. Liu and J. H. Ye, Constructing and controlling of highly dispersed metallic sites for catalysis, Nano Today, 2018, 19, 108-125.

40 X. D. Zhang, D. Wu, X. Shen, P. X. Liu, F. Y. Fan and S. J. Fan, In Vivo Renal Clearance, Biodistribution, Toxicity of Gold Nanoclusters, Biomaterials, 2012, 33, 4628-4638.

41 J. B. Liu, M. X. Yu, C. Zhou, S. Y. Yang, X. H. Ning and J. Zheng, Passive Tumor Targeting of Renal-Clearable Luminescent Gold Nanoparticles: Long Tumor Retention and Fast Normal Tissue Clearance, J. Am. Chem. Soc., 2013, 135(13), 4978-4981.

42 X. R. Song, N. Goswami, H. H. Yang and J. P. Xie, Functionalization of metal nanoclusters for biomedical applications, Analyst, 2016, 141, 3126-3140.

43 Q. F. Yao, X. Yuan, T. K. Chen, D. T. Leong and J. P. Xie, Engineering Functional Metal Materials at the Atomic Level, Adv. Mater., 2018, 30, 1802751.

44 A. A. Herzing, C. J. Kiely, A. F. Carley, P. Landon and G. J. Hutching, Identification of Active Gold Nanoclusters on Iron Oxide Supports for CO Oxidation, Science, 2008, 321, 1331-1335.

45 A. Mathew and T. Pradeep, Noble Metal Clusters: Applications in Energy, Environment, and Biology, Part. Part. Syst. Charact., 2014, 31, 1017-1053.

46 Z. K. Wu, M. A. MacDonald, J. Chen, P. Zhang and R. C. Jin, Kinetic Control and Thermodynamic Selection in the Synthesis of Atomically Precise Gold Nanoclusters, J. Am. Chem. Soc., 2011, 133, 9670-9673.

47 H. Zhang, X. Huang, L. Li, G. W. Zhang, I. Hussain, Z. Li and B. Tan, Photoreductive synthesis of water-soluble fluorescent metal nanoclusters, Chem. Commun., 2012, 48, 567-569.

48 H. Y. Liu, X. Zhang, X. M. Wu, L. P. Jiang, C. Burda and J. J. Zhu, Rapid sonochemical synthesis of highly luminescent non-toxic $\mathrm{Au}$ NCs and $\mathrm{Au}$ @ $\mathrm{Ag}$ NCs and $\mathrm{Cu}$ (II) sensing, Chem. Commun., 2011, 47, 4237-4239.

49 Y. Yue, T. Y. Liu, H. W. Li, Z. Y. Liu and Y. Q. Wu, Microwave-assisted synthesis of BSA-protected small gold nanoclusters and their fluorescence-enhanced sensing of silver(I) ions, Nanoscale, 2012, 4, 2251-2254.

50 W. W. Guo, J. P. Yuan and E. K. Wang, Organic-soluble fluorescent Au8 clusters generated from heterophase ligand-exchange induced etching of gold nanoparticles and their electrochemiluminescence, Chem. Commun., 2012, 48, 3076-3078.

51 M. L. Cui, Y. Zhao and Q. J. Song, Synthesis, optical properties and applications of ultra-small luminescent gold nanoclusters, Trends Anal. Chem., 2014, 57, 73-82.

52 N. Goswami, Q. F. Yao, T. K. Chen and J. P. Xie, Mechanistic exploration and controlled synthesis of precise thiolate-gold nanoclusters, Coord. Chem. Rev., 2016, 329, 1-15.

53 N. K. Chaki, Y. Negishi, H. Tsunoyama, Y. Shichibu and T. Tsukuda, Ubiquitous 8 and 29 kDa Gold: Alkanethiolate
Cluster Compounds: Mass-Spectrometric Determination of Molecular Formulas and Structural Implications, J. Am. Chem. Soc., 2008, 130, 8608-8610.

54 C. A. Fields-Zinna, J. S. Sampson, M. C. Crowe, J. B. Tracy, J. F. Parker, A. M. deNey, D. C. Muddiman and R. W. Murray, Tandem Mass Spectrometry of Thiolate-Protected

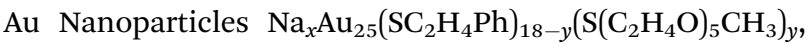
J. Am. Chem. Soc., 2009, 131, 13844-13851.

55 A. Ghosh, T. Udayabhaskararao and T. Pradeep, One-Step Route to Luminescent $\mathrm{Au}_{18} \mathrm{SG}_{14}$ in the Condensed Phase and Its Closed Shell Molecular Ions in the Gas Phase, J. Phys. Chem. Lett., 2012, 3, 1997-2002.

56 Y. Negishi, Y. Takasugi, S. Sato, H. Yao, K. Kimura and T. Tsukuda, Magic-Numbered $\mathrm{Au}_{n}$ Clusters Protected by Glutathione Monolayers $(n=18,21,25,28,32,39)$ : Isolation and Spectroscopic Characterization, J. Am. Chem. Soc., 2004, 126, 6518-6519.

57 E. Porret, L. Sancey, A. Martín-Serrano, M. I. Montañez, R. Seeman, A. Yahia-Ammar, H. Okuno, F. Gomez, A. Ariza, N. Khildebrandt, J. B. Fleury, J. L. Coll and X. Le Guével, Hydrophobicity of Gold Nanoclusters Influences Their Interactions with Biological Barriers, Chem. Mater., 2017, 29, 7497-7506.

58 A. V. Shytov and P. B. Allen, Electronic polarity of nanoclusters: Quantum and many-body effects, Phys. Rev. B: Condens. Matter Mater. Phys., 2006, 74, 075419.

59 M. Z. Zhu, H. F. Qian, X. M. Meng, S. S. Jin, Z. K. Wu and R. C. Jin, Chiral $\mathrm{Au}_{25}$ Nanospheres and Nanorods: Synthesis and Insight into the Origin of Chirality, Nano Lett., 2011, 11, 3963-3969.

60 K. Pyo, V. D. Thanthirige, S. Y. Yoon, G. Ramakrishna and D. Lee, Enhanced luminescence of $\mathrm{Au}_{22}(\mathrm{SG})_{18}$ nanoclusters via rational surface engineering, Nanoscale, 2016, 8, 20008-20016.

61 I. Guryanov, F. Polo, E. V. Ubyvovk, E. Korzhikov-Vlakh, T. Tennikova, A. T. Rad, M. P. Nieh and F. Maran, Polylysine-grafted $\mathrm{Au}_{144}$ nanoclusters: birth and growth of a healthy surface-plasmon-resonance-like band, Chem. Sci., 2017, 8, 3228-3238.

62 A. Dass, P. R. Nimmala, V. R. Jupally and N. Kothalawala, $\mathrm{Au}_{103}(\mathrm{SR})_{45}, \mathrm{Au}_{104}(\mathrm{SR})_{45}, \mathrm{Au}_{104}(\mathrm{SR})_{46}$ and $\mathrm{Au}_{105}(\mathrm{SR})_{46}$ nanoclusters, Nanoscale, 2013, 5, 12082-12085.

63 S. Fedrigo, W. Harbich and J. Buttet, Optical Response of $\mathrm{Ag}_{2}, \mathrm{Ag}_{3}, \mathrm{Au}_{2}$, and $\mathrm{Au}_{3}$ in Argon Matrices, J. Chem. Phys., 1993, 99, 5712-5717.

64 B. A. Collings, K. Athanassenas, D. Lacombe, D. M. Rayner and P. A. Hackett, Optical absorption spectra of $\mathrm{Au}_{7}, \mathrm{Au}_{9}$, $\mathrm{Au}_{11}$, and $\mathrm{Au}_{13}$, and their cations: gold clusters with 6, 7, 8, 9, 10, 11, 12, and 13 s-electrons, J. Chem. Phys., 1994, 101, 3506-3513.

65 J. Zheng, C. W. Zhang and R. M. Dickson, Highly fluorescent, water-soluble, size-tunable gold quantum dots, Phys. Rev. Lett., 2004, 93, 077402.

66 J. Zheng, C. Zhou, M. Yu and J. Liu, Different Sized Luminescent Gold Nanoparticles, Nanoscale, 2012, 4, 4073-4083. 
67 S. Wang, X. Meng, A. Das, T. Li, Y. Song, T. Cao, X. Zhu, M. Zhu and R. Jin, A 200-fold Quantum Yield Boost in the Photoluminescence of Silver-Doped $\mathrm{Ag}_{x} \mathrm{Au}_{25-x}$ Nanoclusters: The 13 th Silver Atom Matters, Angew. Chem., Int. Ed., 2014, 53, 2376-2380.

68 C. M. Andolina, A. C. Dewar, A. M. Smith, L. E. Marbella, M. J. Hartmann and J. E. Millstone, Photoluminescent Gold-Copper Nanoparticle Alloys with CompositionTunable Near-Infrared Emission, J. Am. Chem. Soc., 2013, 135, 5266-5269.

69 Y. X. Chen, C. Liu, Q. Tang, C. J. Zeng, T. Higaki, A. Das, D. E. Jiang, N. L. Rosi and R. C. Jin, Isomerism in $\mathrm{Au}_{28}(\mathrm{SR})_{20}$ Nanocluster and Stable Structures, J. Am. Chem. Soc., 2016, 138, 1482-1485.

70 S. Link, A. Beeby, S. FitzGerald, M. A. El-Sayed, T. G. Schaaff and R. L. Whetten, Visible to Infrared Luminescence from a 28-Atom Gold Cluster, J. Phys. Chem. B, 2002, 106, 3410-3415.

71 S. A. Miller, J. M. Womick, J. F. Parker, R. W. Murray and A. M. Moran, Femtosecond Relaxation Dynamics of $\mathrm{Au}_{25} \mathrm{~L}_{18}{ }^{-}$Monolayer-Protected Clusters, J. Phys. Chem. C, 2009, 113, 9440-9444.

$72 \mathrm{Z}$. Wu and R. Jin, On the Ligand's Role in the Fluorescence of Gold Nanoclusters, Nano Lett., 2010, 10, 2568-2573.

73 J. Liu, H. W. Li, W. X. Wang and Y. Q. Wu, Thermally prepared ultrabright adenosine monophosphate capped gold nanoclusters and the intrinsic mechanism, J. Mater. Chem. B, 2017, 5, 3550-3556.

74 G. L. Wang, L. Y. Jin and Y. M. Dong, Intrinsic enzyme mimicking activity of gold nanoclusters upon visible light triggering and its application for colorimetric trypsin detection, Biosens. Bioelectron., 2015, 64, 523-529.

75 C. X. Chen, D. Zhao, Y. Y. Jiang, P. J. Ni, C. H. Zhang, B. Wang, F. Yang, Y. Z. Lu and J. Sun, Logically Regulating Peroxidase-Like Activity of Gold Nanoclusters for Sensing Phosphate-Containing Metabolites and Alkaline Phosphatase Activity, Anal. Chem., 2019, 91, 15017-15024.

76 S. Chakraborty, S. Babanova, R. C. Rocha, A. Desireddy, K. Artyushkova, A. E. Boncella, P. Atanassov and J. S. Martinez, A Hybrid DNA-Templated Gold Nanocluster For Enhanced Enzymatic Reduction of Oxygen, J. Am. Chem. Soc., 2015, 137, 11678-11687.

77 D. J. Mikolajczak, A. A. Berger and B. Koksch, Catalytically Active Peptide-Gold Nanoparticle Conjugates: Prospecting for Artificial Enzymes, Angew. Chem., Int. Ed., 2020, 59, 8776-8785.

78 W. Y. Chen, H. Y. Chang, J. K. Lu, Y. C. Huang, S. G. Harroun, Y. T. Tseng, Y. J. Li, C. C. Huang and H. T. Chang, Self-Assembly of Antimicrobial Peptides on Gold Nanodots: Against Multidrug-Resistant Bacteria and Wound-Healing Application, Adv. Funct. Mater., 2015, 25, 7189-7199.

79 K. Pyo, N. H. Ly, S. Y. Yoon, Y. M. Shen, S. Y. Choi, S. Y. Lee, S. W. Joo and D. Lee, Highly Luminescent FolateFunctionalized $\mathrm{Au}_{22}$ Nanoclusters for Bioimaging, Adv. Healthcare Mater., 2017, 6, 1700203.

80 Z. M. Liu, L. Turyanska, F. Zamberlan, S. Pacifico, T. D. Bradshaw, F. Moro, M. W. Fay, H. E. L. Williams and
N. R. Thomas, Synthesis of folic acid functionalized gold nanoclusters for targeting folate receptor-positive cells, Nanotechnology, 2019, 30, 505102.

81 H. Y. Chen, B. W. Li, X. Y. Ren, S. N. Li, Y. X. Ma, S. S. Cui and Y. Q. Gu, Multifunctional near-infrared-emitting nanoconjugates based on gold clusters for tumor imaging and therapy, Biomaterials, 2012, 33, 8461-8476.

82 M. J. Wang, C. Y. Sun, L. Y. Wang, X. H. Ji, Y. B. Bai, T. J. Li and J. H. Li, Electrochemical Detection of DNA Immobilized on Gold Colloid Particles Modified Self-assembled Monolayer Electrode with Silver Nanoparticle Label, J. Pharm. Biomed. Anal., 2003, 33, 1117-1125.

83 Y. Negishi, K. Nobusada and T. Tsukuda, GlutathioneProtected Gold Clusters Revisited: Bridging the Gap between Gold(I)-Thiolate Complexes and Thiolate-Protected Gold Nanocrystals, J. Am. Chem. Soc., 2005, 127, 5261-5270.

84 J. Nishigaki, R. Tsunoyama, H. Tsunoyama, N. Ichikuni, S. Yamazoe, Y. Negishi, M. Ito, T. Matsuo, K. Tamao and T. Tsukuda, A New Binding Motif of Sterically Demanding Thiolates on a Gold Cluster, J. Am. Chem. Soc., 2012, 134, 14295-14297.

85 L. C. McKenzie, T. O. Zaikova and J. E. Hutchison, Structurally Similar Triphenylphosphine-Stabilized Undecagolds, $\mathrm{Au}_{11}\left(\mathrm{PPh}_{3}\right)_{7} \mathrm{Cl}_{3}$ and $\left[\mathrm{Au}_{11}\left(\mathrm{PPh}_{3}\right)_{8} \mathrm{Cl}_{2}\right] \mathrm{Cl}$, Exhibit Distinct Ligand Exchange Pathways with Glutathione, J. Am. Chem. Soc., 2014, 136, 13426-13435.

86 X. K. Wan, Q. Tang, S. F. Yuan, D. Jiang and Q. M. Wang, $\mathrm{Au}_{19}$ Nanocluster Featuring a V-Shaped Alkynyl-Gold Motif, J. Am. Chem. Soc., 2015, 137, 652-655.

87 Y. Y. Zhao, Z. L. Chen, Y. F. Chen, J. Xu, J. H. Li and $\mathrm{X}$. Y. Jiang, Synergy of nonantibiotic drugs and pyrimidinethiol on gold nanoparticles against superbugs, J. Am. Chem. Soc., 2013, 135, 12940-12943.

88 N. Q. Gong, X. W. Ma, X. X. Ye, Q. F. Zhou, X. L. Tan, S. K. Yao, S. D. Huo, T. B. Zhang, S. Z. Chen, X. C. Teng, X. X. Hu, J. Yu, Y. L. Gan, H. D. Jiang, J. H. Li and X. J. Liang, Carbon dot-supported atomically-dispersed gold as a mitochondrial oxidative stress amplifier for cancer treatment, Nat. Nanotechnol., 2019, 14, 379-387.

89 J. Zheng, J. T. Petty and R. M. Dickson, High Quantum Yield Blue Emission from Water-Soluble $\mathrm{Au}_{8}$ Nanodots, J. Am. Chem. Soc., 2003, 125, 7780-7781.

90 D. Y. An, J. G. Su, J. K. Weber, X. Y. Gao, R. H. Zhou and J. Y. Li, A Peptide-Coated Gold Nanocluster Exhibits Unique Behavior in Protein Activity Inhibition, J. Am. Chem. Soc., 2015, 137, 8412-8418.

91 Z. P. Wang, J. Q. Hu, Y. Jin, X. Yao and J. H. Li, In Situ Amplified Chemiluminescent Detection of DNA and Immunoassay of IgG Using Special-Shaped Gold Nanoparticles as Label, Clin. Chem., 2006, 52, 1958-1961.

92 O. Varnavski, G. Ramakrishna, J. Kim, D. Lee and T. Goodson, Critical Size for the Observation of Quantum Confinement in Optically Excited Gold Clusters, J. Am. Chem. Soc., 2010, 132, 16-17.

93 D. Hu, Z. H. Sheng, P. Gong, P. F. Zhang and L. T. Cai, Highly selective fluorescent sensors for $\mathrm{Hg}^{2+}$ based on 
bovine serum albumin-capped gold nanoclusters, Analyst, 2010, 135, 1411-1416.

94 H. Wei, Z. D. Wang, L. M. Yang, S. L. Tian, C. J. Hou and Y. Lu, Lysozyme-stabilized gold fluorescent cluster: Synthesis and application as $\mathrm{Hg}^{2+}$ sensor, Analyst, 2010, 135, 1406-1410.

95 X. L. Guével, N. Daum and M. Schneider, Synthesis and characterization of human transferrin-stabilized gold nanoclusters, Nanotechnology, 2011, 22, 275103.

96 P. L. Xavier, K. Chaudhari, P. K. Verma, S. K. Pal and T. Pradeep, Luminescent quantum clusters of gold in transferrin family protein, lactoferrin exhibiting FRET, Nanoscale, 2010, 2, 2769-2776.

97 H. Kawasaki, K. Yoshimura, K. Hamaguchi and R. Arakawa, Trypsin-stabilized fluorescent gold nanocluster for sensitive and selective $\mathrm{Hg}^{2+}$ detection, Anal. Sci., 2011, 27, 591-596.

98 F. Wen, Y. H. Dong, L. Feng, S. Wang, S. C. Zhang and X. R. Zhang, Horseradish peroxidase functionalized fluorescent gold nanoclusters for hydrogen peroxide sensing, Anal. Chem., 2011, 83, 1193-1196.

99 J. J. Luo, A. Rasooly, L. Q. Wang, K. Zeng, C. C. Shen, P. Qian, M. H. Yang and F. Qu, Fluorescent turn-on determination of the activity of peptidases using peptide templated gold nanoclusters, Microchim. Acta, 2016, 183, 605-610.

100 N. Q. Gong, X. C. Teng, J. H. Li and X. J. Liang, Antisense Oligonucleotide-Conjugated Nanostructure-Targeting lncRNA MALAT1 Inhibits Cancer Metastasis, ACS Appl. Mater. Interfaces, 2019, 11, 37-42.

101 T. A. C. Kennedy, J. L. MacLean and J. W. Liu, Blue emitting gold nanoclusters templated by poly-cytosine DNA at low $\mathrm{pH}$ and poly-adenine DNA at neutral pH, Chem. Commun., 2012, 48, 6845-6847.
102 R. C. Triulzi, M. Micic, S. Giordani, M. Serry, W. A. Chiou and R. M. Leblanc, Immunoasssay based on the Antibodyconjugated PAMAM-dendrimer-gold Quantum dot Complex, Chem. Commun., 2006, 5068-5070.

103 G. H. Yang, J. J. Shi, S. Wang, W. W. Xiong, L. P. Jiang, C. Burda and J. J. Zhu, Fabrication of a Boron Nitride-gold Nanocluster Composite and its Versatile Application for Immunoassays, Chem. Commun., 2013, 49, 10757-10759.

104 Y. Zhang, C. Y. Zhang, C. Xu, X. L. Wang, C. Liu, G. I. N. Waterhouse, Y. L. Wang and H. Z. Yin, Ultrasmall Au nanoclusters for biomedical and biosensing applications: A mini-review, Talanta, 2019, 200, 432-442.

105 T. P. Silverstein, Marcus Theory: Thermodynamics Can Control the Kinetics of Electron Transfer Reactions, J. Chem. Educ., 2012, 89, 1159-1167.

106 J. L. Brennan, M. R. Branham, J. F. Hicks, A. J. Osisek, R. L. Donkers, D. G. Georganopoulou and R. W. Murray, Electron Hopping Dynamics in Monolayer-Protected Au Cluster Network Polymer Films by Rotated Disk Electrode Voltammetry, Anal. Chem., 2004, 76, 5611-5619.

107 J. Kim and D. Lee, Electron Hopping Dynamics in Au38 Nanoparticle Langmuir Monolayers at the Air/Water Interface, J. Am. Chem. Soc., 2006, 128, 4518-4519.

108 J. A. Scholl, A. García-Etxarri, A. L. Koh and J. A. Dionne, Observation of Quantum Tunneling between Two Plasmonic Nanoparticles, Nano Lett., 2013, 13, 564-569.

109 A. Yu, S. W. Li, G. Czap and W. Ho, Tunneling-ElectronInduced Light Emission from Single Gold Nanoclusters, Nano Lett., 2016, 16, 5433-5436.

110 H. J. Xiang, W. Feng and Y. Chen, Single-Atom Catalysts in Catalytic Biomedicine, Adv. Mater., 2020, 1905994. 